\title{
Nachgefragt
}

\section{Pflege muss eigenständig werden}

\author{
Die Bunderegierung arbeitet zurzeit an einem neuen Pflegeberufege- \\ setz, mit dem die Pflegeausbildung modernisiert und ein einheitliches, \\ attraktiveres Berufsbild für alle Pflegeberufe geschaffen werden soll. \\ Werden mit der Etablierung dieses Gesetzes die Probleme, die es in der \\ Pflege gegenwärtig gibt, gelöst werden? Wir fragten den Pflegebevoll- \\ mächtigten der Bundesregierung, Staatssekretär Karl-Josef Laumann.
}

? Herr Laumann, warum wollen Sie den Beruf der Kranken- und Altenpflege zusammenführen?

Laumann: Wir wollen in dieser Wahlperiode zu einer Generalisierung der Pflegeausbildung kommen. So steht es auch im Koalitionsvertrag, dem alle Minister auf Landesebene zugestimmt haben. Ich hoffe, dass sich u.a. dadurch die Pflege in Deutschland mehr emanzipiert. Der Beruf kann nur attraktiv werden, wenn er zu einem eigenständigen Teil des deutschen Gesundheitswesens wird. Das Zusammenführen der Alten- und Krankenpflege ist hierzu ein wichtiger Schritt. Bund und Länder haben sich in fast allen Punkten geeinigt. Geklärt werden müssen nur noch Details, etwa, wie wir künftig die Pflegeschulen finanzieren.

\section{? Haben wir genug Nachwuchs für die} Pflege?

Laumann: Die größte Herausforderung ist es, die Menschen zu finden, die in den Pflegeberuf wollen. Am Beispiel der Altenpflege wird es deutlich: Dort haben wir rund 700.000 Pflegekräfte und jedes Jahr wächst der Bedarf um etwa 2-3\%. Das sind über 20.000 zusätzliche Stellen. Der Arbeitsmarkt für Pflegekräfte ist aber derzeit ziemlich leergefegt - es ist teilweise sehr schwer, geeignetes Personal zu finden. Umso wichtiger ist daher für jeden von uns das private Umfeld. Das, was Familien aus Liebe zu ihren Angehörigen leisten, können wir nicht komplett durch professionelle Kräfte ersetzen.

? Und wie steht es aktuell um die Pflege in unseren Krankenhäusern?
Laumann: Die gesetzlichen Krankenkassen haben den Auftrag, Medizin, Pflege und ärztliche Leistung zu bezahlen. Die Landesregierungen sind für die Investitionen in den Krankenhäusern verantwortlich. Doch dieser Verantwortung kommen sie nur unzureichend nach: Die entsprechenden Fördermittel sind von rund 3,9 Milliarden Euro im Jahr 1993 auf rund 2,7 Milliarden Euro 2013 zurückgegangen. Das hat dazu geführt, dass viele Krankenhäuser nun das Geld der Krankenkassen nehmen und es in die Gebäudeunterhaltung anstatt in die Pflege stecken. Deswegen kommt es zu Personalengpässen in den Krankenhäusern. Und deshalb sind auch die Pflegekräfte der Charité auf die Straße gegangen - die wollten nicht mehr Geld, sondern mehr Personal.

? Wie können wir ganz generell die $\mathrm{Zu}$ kunft in der Pflege meistern?

Laumann: Die große Herausforderung kommt erst noch auf uns zu, wenn die geburtenstarken Jahrgänge der Nachkriegszeit, die Baby-Boomer, in 20 Jahren pflegebedürftig sein werden und gleichzeitig die geburtenschwachen Jahrgänge die Schulen verlassen. Für die Grund- und Behandlungspflege werden wir dann das Personal voraussichtlich haben. Aber das, was Menschen an Gesprächen und Empathie im Krankheitsfall auch brauchen, das werden wir niemals allein über den Staat organisieren können. Dafür wird es schlichtweg nicht genügend Personal geben. Weil wir es nicht bezahlen können und, weil wir die Ressourcen an Nachwuchskräften gar nicht haben.

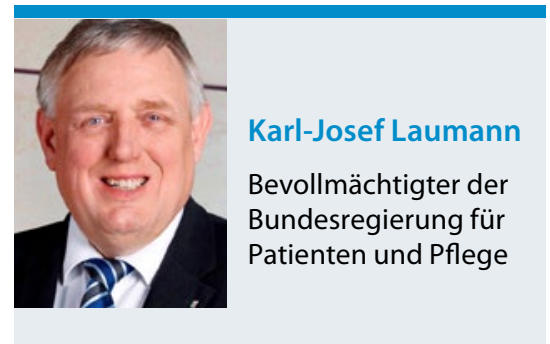

? Sie entbürokratisieren die Pflege, wie sieht das konkret aus?

Laumann: Die Grundidee ist bestechend einfach. Nur wenn etwas vom normalen Pflegealltag abweicht, muss das noch aufgeschrieben werden. Das heißt: Pflegekräfte schreiben nicht mehr auf, dass ein Pflegebedürftiger kein Fieber hat, sondern "nur" noch, wenn er Fieber oder andere Auffälligkeiten hat. Die neue Dokumentation vertraut den Pflegekräften, dass sie die Abweichungen registrieren und dokumentieren. So wissen die Pflegekräfte nach dem Schichtwechsel, worauf sie besonders achten - und sich kümmern müssen.

? Wie überzeugen Sie die Pflegenden von Ihrer Idee?

Laumann: Wir haben 650 Multiplikatoren geschult, die nun in den Einrichtungen die Idee und das neue Modell der Entbürokratisierung dem dortigen Personal erklären. Das soll dann wie ein Schneeballsystem immer weiter getragen werden. Wir gehen davon aus, dass wir damit bis zum Jahresende gut ein Viertel der Einrichtungen überzeugen. Klappt das, wäre das ein Meilenstein.

Das Interview führte Holger Senft. 